\title{
Particle production in the interiors of acoustic black holes
}

\author{
Roberto Balbinot* \\ Dipartimento di Fisica dell'Università di Bologna and INFN sezione di Bologna, \\ Via Irnerio 46, 40126 Bologna, Italy \\ and Centro Fermi-Museo Storico della Fisica e Centro Studi e Ricerche Enrico Fermi, \\ Piazza del Viminale 1, 00184 Roma, Italy \\ Alessandro Fabbri ${ }^{\dagger}$ \\ Departamento de Física Teórica and IFIC, Universidad de Valencia-CSIC, \\ Calle Dr. Moliner 50, 46100 Burjassot, Spain; \\ Centro Fermi-Museo Storico della Fisica e Centro Studi e Ricerche Enrico Fermi, \\ Piazza del Viminale 1, 00184 Roma, Italy \\ and Laboratoire de Physique Théorique, CNRS UMR 8627, Bâtiment 210, \\ Université Paris-Sud 11, 91405 Orsay Cedex, France \\ Richard A. Dudley $\odot^{\ddagger}$ and Paul R. Anderson $\odot^{\S}$ \\ Department of Physics, Wake Forest University, Winston-Salem, North Carolina 27109, USA
}

(Received 15 October 2019; published 27 November 2019)

\begin{abstract}
Phonon creation inside the horizons of acoustic black holes is investigated using two simple toy models. It is shown that, unlike what occurs in the exterior regions, the spectrum is not thermal. This nonthermality is due to the anomalous scattering that occurs in the interior regions.
\end{abstract}

DOI: 10.1103/PhysRevD.100.105021

\section{INTRODUCTION}

Among the many spectacular celestial objects that populate our Universe, black holes (BHs) are perhaps the most intriguing. The presence of a causal horizon prevents any direct observation of the interior. According to theoretical studies based on general relativity, the interior is a place where peculiar physical effects occur, which cannot be confirmed by astronomical observations. The situation has positively changed in recent times with the advent of so-called "analog BHs" [1,2]. These are condensed matter systems that are realizable in the laboratory, which mimic some of the essential features of gravitational BHs. A typical example is a Bose-Einstein condensate (BEC) fluid (see e.g., [3]) whose flow becomes supersonic [4-6]. The supersonic region, trapping sound waves inside it, is the analog of the BH interior. The sonic surface, where the speed of the flow equals the local speed of sound, plays the role of the horizon. This sonic horizon however has no causal significance at all: there is nothing to prevent one from directly observing the interior region. Indeed the first experimental observations of the analog of Hawking

\footnotetext{
*balbinot@bo.infn.it †afabbri@ific.uv.es \#dudlra13@wfu.edu §anderson@wfu.edu
}

radiation [7] in BECs by Steinhauer et al. [8,9] were made by performing simultaneous measurements of the density outside and inside the sonic horizon. A peak was observed in the resulting in-out density-density correlation function that was predicted in $[10,11]$ and which is the "smoking gun" signaling the presence of Hawking radiation. In the same spirit, one can imagine that other processes that are predicted to take place in the interior of a $\mathrm{BH}$ can be experimentally verified by looking at appropriate analog models.

With this as motivation, in this paper we discuss the unusual features of scattering by a potential inside the horizon of a stationary BEC analog $\mathrm{BH}$ and its consequences. The calculations are done in the analog spacetime using quantum field theory in curved space techniques. These are the same types of calculations that one would do to explore similar effects in the interior of a real black hole.

In quantum mechanics in the presence of a potential, an incident flux is split into a transmitted and a reflected part (see Fig. 1). Reflection $(R)$ and transmission $(T)$ coefficients satisfy the unitary relation $|R|^{2}+|T|^{2}=1$, which is the conservation of probability. Note that the previous relation implies that $|R|^{2}$ and $|T|^{2} \leq 1$.

Inside the horizon of a $\mathrm{BH}$ both the transmitted and the "would be reflected" part of the field are forced to propagate in the same direction, namely toward the center of the black hole (see Fig. 2). The scattering is "anomalous" 


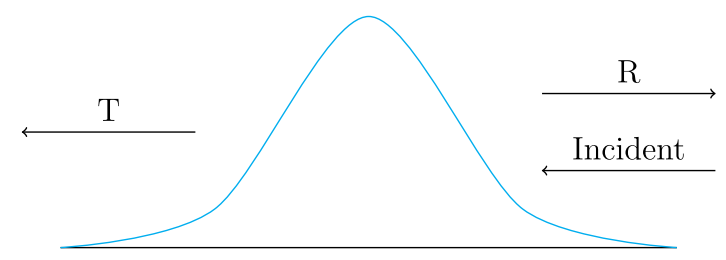

FIG. 1. Illustration of a plane wave incident onto a potential from the right, which then is partially transmitted to the left and also partial reflected back to the right of the barrier.

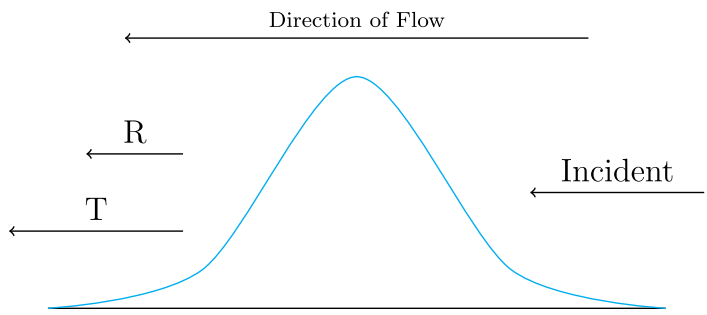

FIG. 2. Illustration of a plane wave incident onto a potential from the right in the interior of a $\mathrm{BH}$, which then is partially transmitted to the left and also partially reflected; however, the reflected portion is also moving to the left of the barrier, since in the interior the wave is forced to travel further into the $\mathrm{BH}$.

and $R$ and $T$ no longer satisfy the previous unitary relation. Instead they satisfy $|T|^{2}-|R|^{2}=1$ which implies particle creation since $|T|^{2} \geq 1$. Another way to think about this is that, while the outside region of a nonrotating black hole is static, the interior can be thought of as a dynamical cosmology in which particle creation occurs.

We shall deal with both massless and massive quantum fields. For the latter case there is usually a mass gap, namely $E \geq m$, where $E$ is the conserved (Killing) energy and $m$ is the mass of the particle. Inside a BH the former inequality no longer holds, and $E$ can take any value, even negative ones.

In Sec. II a brief review is given of the setup for BEC analog black holes. In Sec. III particle production is investigated in the case of massless phonons with a double delta function potential. In Sec. IV particle production is investigated for massive phonons when the effective potential is zero and the mass term in the mode equation is approximated by two step functions. Section V contains a discussion of our results and comparisons with some previous work.

\section{THE SETTING}

Under the hydrodynamic approximation the phase fluctuation operator $\hat{\phi}$ in a BEC satisfies a covariant version of d'Alembert's wave equation (see for instance [2])

$$
\hat{\square} \hat{\phi}=0,
$$

where $\hat{\square}=\hat{\nabla}_{\mu} \hat{\nabla}^{\nu}$ is evaluated on a fictitious curved spacetime metric, called the acoustic metric, which in our case we write as follows:

$d s^{2}=\frac{n}{m_{a} c}\left[-c^{2}(x) d T^{2}+\left(d x+v_{0} d T\right)^{2}+d y^{2}+d z^{2}\right]$,

where $n$ is the density of the condensate (here assumed to be constant), $m_{a}$ is the mass of a single atom of the BEC, and $c(x)$ is the sound speed. The flow is assumed to be stationary and one dimensional along the $x$ axis with the velocity $\vec{v}=-v_{o} \hat{x}$ constant and directed from right to left.

For a typical profile used in BEC analog models $c(x)$ becomes constant in both asymptotic regions $(x \rightarrow \pm \infty)$ so that $\lim _{x \rightarrow+\infty} c(x)=c_{r}$ and $\lim _{x \rightarrow-\infty} c(x)=c_{l}$ with $c_{r}>$ $v_{0}$ and $c_{l}<v_{0}$. Thus the asymptotic regions are homogeneous and the profile monotonically decreases from right to left. The profile $c(x)$ is chosen so that the horizon $c(x)=$ $v_{0}$ is at $x=0$. In the region $x<0$, where $c(x)<v_{0}$, the metric describes the interior region of the acoustic $\mathrm{BH}$, while for $x>0$, where $c(x)>v_{0}$, the metric describes the exterior region of the acoustic $\mathrm{BH}$. We call the exterior the $r$ region and the interior the $l$ region. Performing a dimensional reduction along the transverse direction and passing from the Gullstrand-Painleve coordinates $(T, x)$ to the Schwarzschild-like ones $\left(t, x^{*}\right)$ via the transformation

$t=T-\int d x \frac{v_{0}}{c(x)^{2}-v_{0}^{2}} \quad$ and $\quad x^{*}=\int d x \frac{c(x)}{c(x)^{2}-v_{0}^{2}}$,

the wave equation (2.1) can be reduced to

$$
\left[-\partial_{t}^{2}+\partial_{x^{*}}^{2}-k_{\perp}^{2}\left(c^{2}-v_{0}^{2}\right)+V_{\mathrm{eff}}\right] \hat{\phi}^{(2)}=0,
$$

where the effective potential is given by

$V_{\mathrm{eff}} \equiv \frac{c^{2}-v_{0}^{2}}{c}\left[\frac{1}{2} \frac{d^{2} c}{d x^{2}}\left(1-\frac{v_{0}^{2}}{c^{2}}\right)-\frac{1}{4 c}\left(\frac{d c}{d x}\right)^{2}+\frac{5 v_{0}^{2}}{4 c^{3}}\left(\frac{d c}{d x}\right)^{2}\right]$.

The coefficient $k_{\perp}^{2}$ is related to the transverse momentum, and $\hat{\phi}^{(2)}$ is the dimensionally reduced field operator (see the Appendix of Ref. [12] for details). The last two terms in Eq. (2.4), the masslike term and $V_{\text {eff }}$, cause scattering of the modes. Note that both of these terms vanish at the horizon. There the modes are effectively massless and propagate freely. The second coordinate transformation in Eq. (2.3) maps the $(0,+\infty)$ interval in $x$ in the $r$ region to $(-\infty,+\infty)$ in $x^{*}$, while in $l$ the interval $(-\infty, 0)$ in $x$ is mapped to $(+\infty,-\infty)$ in $x^{*}$ as seen in Fig. 3. According to the standard procedure of quantum field theory in curved 


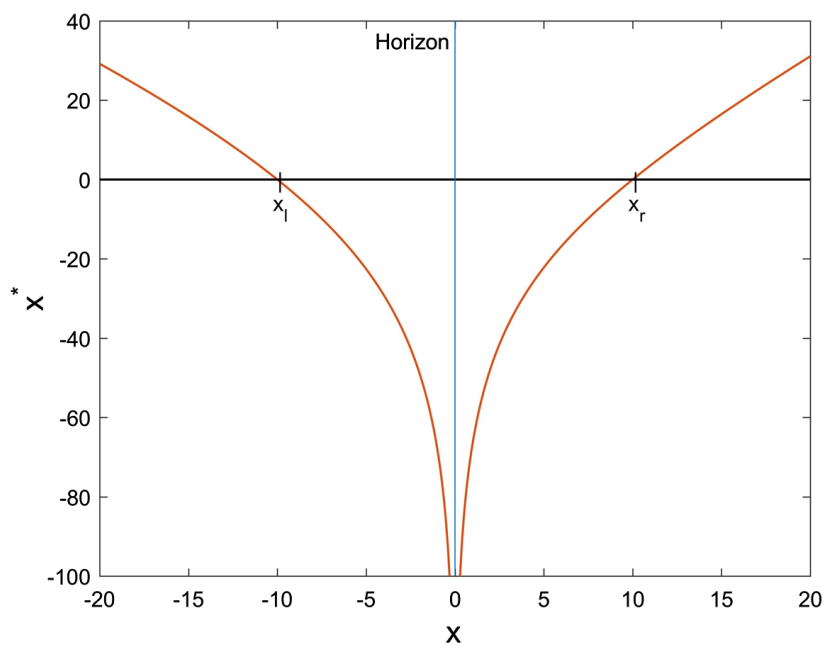

FIG. 3. $x^{*}$ is plotted as a function of $x$ in the interior and exterior regions of the $\mathrm{BH}$. The values of $x$ where $x^{*}=0$ in the $l$ and $r$ regions are labeled as $x_{l}$ and $x_{r}$, respectively. The horizon occurs at $x=0$.

spacetime, the field operator $\hat{\phi}^{(2)}$ is expanded in terms of a complete set of basis functions $\left\{f_{\omega}, f_{\omega}^{*}\right\}$, which are solutions of the classical counterpart of the operator equation (2.4) with the result

$$
\hat{\phi}^{(2)}=\int_{0}^{\infty} d \omega\left(\hat{a}_{\omega} f_{\omega}+\hat{a}_{\omega}^{\dagger} f_{\omega}^{*}\right) .
$$

The creation and annihilation operators $\hat{a}_{\omega}$ and $\hat{a}_{\omega}^{\dagger}$ satisfy the usual commutation relations. The modes $f_{\omega}$ are normalized using the conserved scalar product

$$
\left(f_{\omega}, f_{\omega^{\prime}}\right)=-i \int d \Sigma^{\mu} f_{\omega} \stackrel{\leftrightarrow}{\partial_{\mu}} f_{\omega^{\prime}}^{*}\left[g_{\Sigma}(x)\right]^{1 / 2}
$$

with $d \Sigma^{\mu}=n^{\mu} d \Sigma$, where $\Sigma$ is a Cauchy surface, $n^{\mu}$ a future directed unit vector perpendicular to $\Sigma$, and $g_{\Sigma}$ the determinant of the induced metric. Writing

$$
f_{\omega}=e^{ \pm i \omega t} \chi_{\omega}\left(x^{*}\right)
$$

and substituting into (2.4) gives

$$
\frac{d^{2} \chi_{\omega}}{d x^{* 2}}+\left(\omega^{2}-k_{\perp}^{2}\left(c^{2}-v_{0}^{2}\right)+V_{\mathrm{eff}}\right) \chi_{\omega}=0
$$

In this paper we consider two toy models for the terms in Eq. (2.9) responsible for the scattering which have the advantage of being exactly solvable while, despite their crudeness, encoding all of the basic features of the process we wish to discuss.

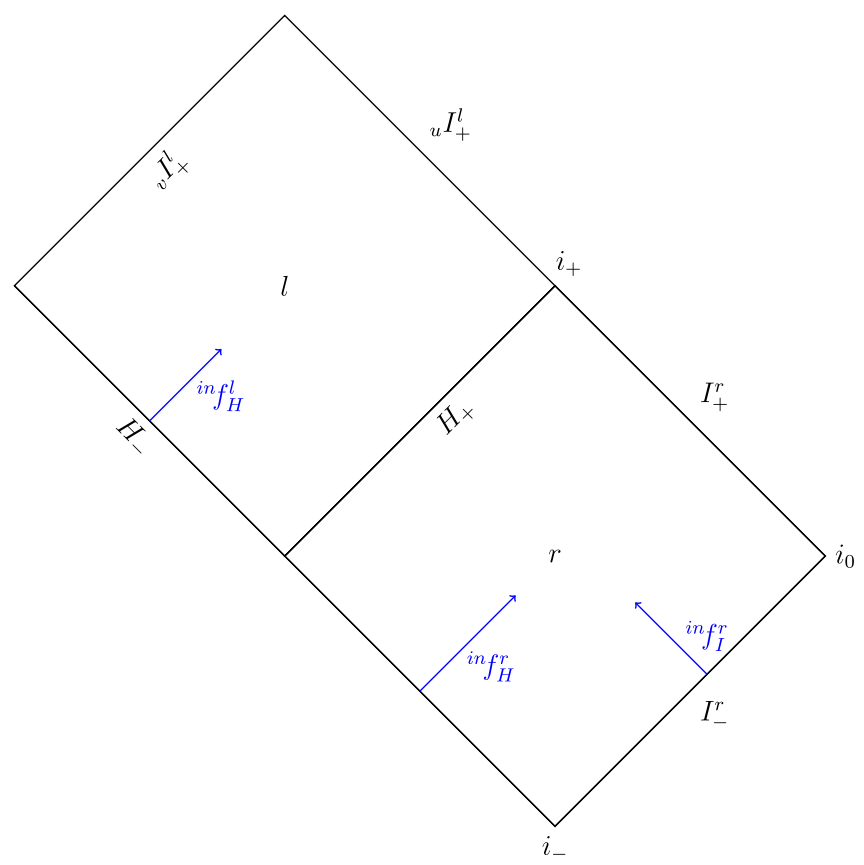

FIG. 4. Penrose diagram with the "in" mode basis schematically illustrated in the $l$ and $r$ regions.

\section{DIRAC DELTA FUNCTION POTENTIALS}

In the first toy model, the transverse excitations are neglected (i.e., $k_{\perp}=0$ ), and $V_{\text {eff }}$ is approximated by two Dirac delta functions, one in region $r$ and one in region $l$. For simplicity we choose them at $x_{r}^{*}=0$ in $r$ and at $x_{l}^{*}=0$ (see Fig. 3) in $l$ leading to ${ }^{1}$

$$
V_{\text {eff }}= \begin{cases}V_{l} \delta\left(x^{*}\right), & x<0 \\ V_{r} \delta\left(x^{*}\right), & x>0 .\end{cases}
$$

The Penrose diagram for the BH metric given in Eq. (2.2) is shown in Fig. 4, where the modes representing our in basis are schematically indicated.

The asymptotic behaviors of these modes are

$$
{ }^{\text {in }} f_{I}^{r}=\frac{e^{-i \omega t} e^{-i \omega x^{*}}}{\sqrt{4 \pi \omega}}=\frac{e^{-i \omega v}}{\sqrt{4 \pi \omega}}
$$

on past null infinity $I_{-}^{r}$;

$$
{ }^{\text {in }} f_{H}^{r}=\frac{e^{-i \omega t} e^{i \omega x^{*}}}{\sqrt{4 \pi \omega}}=\frac{e^{-i \omega u}}{\sqrt{4 \pi \omega}}
$$

on the portion of the past horizon in region $r, H_{-}^{r}$; and

\footnotetext{
${ }^{1}$ For typical flows discussed in the literature which mimic the experimental setup in Refs. [8,9] the effective potential in the interior is dominated by a negative peak. Thus, while our analytic results are valid for arbitrary values of $V_{l}$, when plotting the results we restrict our attention to the case $V_{l}<0$.
} 


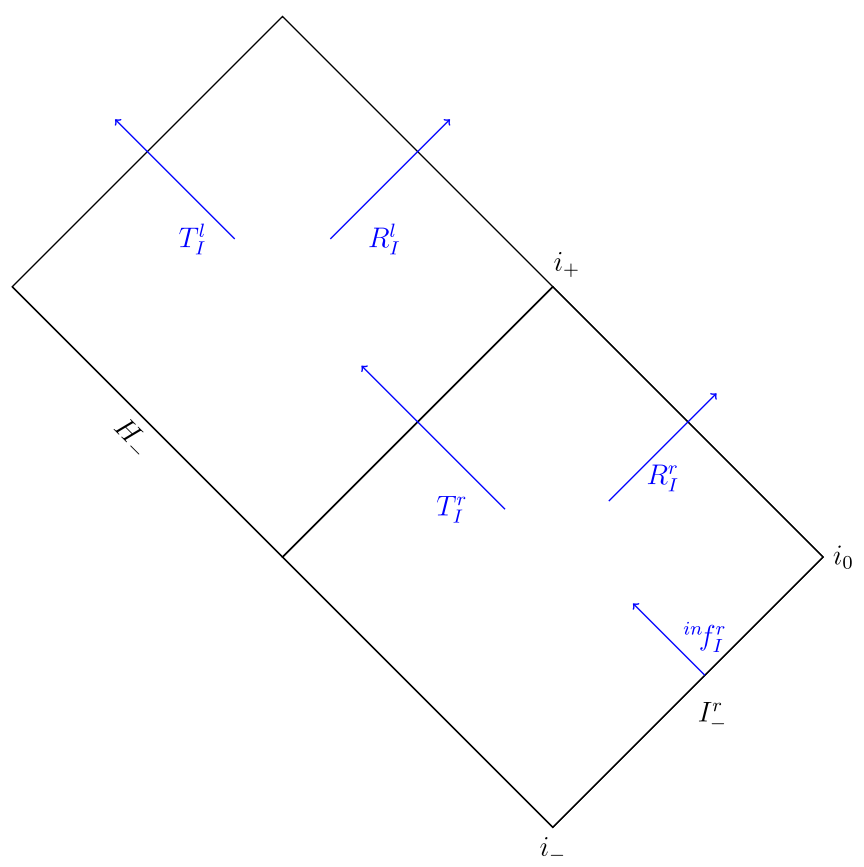

FIG. 5. Penrose diagram illustrating the scattering of an in $f_{I}^{r}$ mode in the $l$ and $r$ regions.

$$
{ }^{i n} f_{H}^{l}=\frac{e^{i \omega t} e^{-i \omega x^{*}}}{\sqrt{4 \pi \omega}}=\frac{e^{i \omega u}}{\sqrt{4 \pi \omega}}
$$

on the portion of the past horizon in region $l, H_{-}^{l}$. These are positive norm modes on $I_{-}^{r}$ or $H_{-}$which together form a Cauchy surface for the spacetime. These modes are associated with annihilation operators in the expansion of the field $\hat{\phi}^{(2)}$ in Eq. (2.6).

In Eqs. (3.2)-(3.4), $u=t-x^{*}$ and $v=t+x^{*}$ are the Eddington-Finkelstein retarded and advanced null coordinates, respectively. Note the + sign in the exponent of Eq. (3.4). The conserved (Killing) energy associated with it is negative and corresponds to excitations called "partners." We need to find the explicit forms of the modes throughout the spacetime.

Let us begin with the in $f_{I}^{r}$ mode whose evolution is represented schematically in Fig. 5. The incoming $v$ mode of the form Eq. (3.2) coming from $I_{-}^{r}$ is partially transmitted $\left(T_{I}^{r}\right)$ toward the horizon as a $v$ mode and partially reflected $\left(R_{I}^{r}\right)$ back to infinity $I_{+}^{r}$ as a $u$ mode by the delta function potential located at $x_{r}^{*}=0$ (see Fig. 6). The

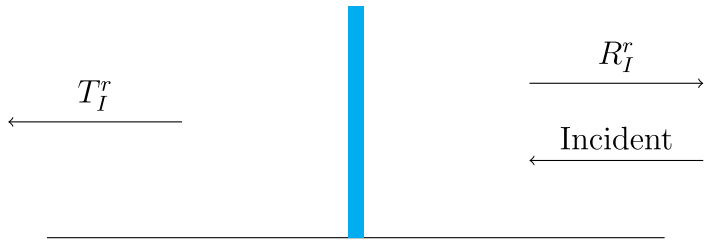

FIG. 6. Illustration of a plane wave incident onto a potential from the right, which then is partially transmitted to the left and also partial reflected back to the right of the barrier.

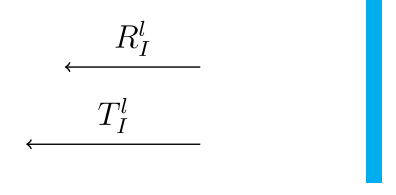

Incident

FIG. 7. Illustration of a plane wave incident onto a negative potential from the right. As this is in the interior of the $\mathrm{BH}$ both the transmitted and reflected portions of the mode are forced to travel further into the $\mathrm{BH}$.

transmitted part crosses the horizon, enters the black hole and is split by the second delta function potential, located inside the black hole at $x_{l}^{*}=0$ (see Fig. 7), into a transmitted $\left(T_{I}^{l}\right) v$ mode and a "reflected" $\left(R_{I}^{l}\right) u$ mode, with both traveling inside along the flow toward left future infinity $\left(I_{+}^{l}\right)$. Thus in the $r$ region

$$
\begin{aligned}
{ }^{\text {in }} f_{I}^{r} & =\frac{e^{-i \omega t}}{\sqrt{4 \pi \omega}}\left[e^{-i \omega x^{*}}+R_{I}^{r} e^{i \omega x^{*}}\right], \quad x^{*}>x_{r}^{*}=0, \\
& =\frac{e^{-i \omega t}}{\sqrt{4 \pi \omega}} T_{I}^{r} e^{-i \omega x^{*}}, \quad x^{*}<x_{r}^{*}=0,
\end{aligned}
$$

and in the $\ell$ region

$$
\begin{aligned}
{ }_{\text {in } f_{I}^{r}}^{r} & =\frac{e^{-i \omega t}}{\sqrt{4 \pi \omega}} T_{I}^{r} e^{-i \omega x^{*}}, \quad x^{*}<x_{\ell}^{*}=0, \\
& =\frac{e^{-i \omega t}}{\sqrt{4 \pi \omega}}\left[T_{I}^{\ell} e^{-i \omega x^{*}}+R_{I}^{\ell} e^{i \omega x^{*}}\right], \quad x^{*}>x_{l}^{*}=0 .
\end{aligned}
$$

The transmission and reflection coefficients are found by matching these solutions across the delta function potentials. In general for a potential of the form $V=\lambda \delta\left(x^{*}\right)$ we require that $\chi\left(x^{*}\right)$ satisfies

$$
\begin{gathered}
\left.\chi\right|_{-}=\left.\chi\right|_{+}, \\
\left.\chi^{\prime}\right|_{+}-\left.\chi^{\prime}\right|_{-}=-\left.\lambda \chi\right|_{-},
\end{gathered}
$$

where $\left.\chi\right|_{ \pm}=\lim _{x^{*} \rightarrow 0^{ \pm}} \chi$ and $\chi^{\prime}$ represents the derivative with respect to $x^{*}$. The results for $\chi_{I}^{r}$ are

$$
\begin{aligned}
T_{I}^{r} & =\frac{\frac{2 i \omega}{V_{r}}}{\frac{2 i \omega}{V_{r}}-1}, \\
R_{I}^{r} & =\frac{1}{\frac{2 i \omega}{V_{r}}-1}, \\
R_{I}^{l} & =\frac{V_{l}}{2 i \omega} T_{I}^{r}, \\
T_{I}^{l} & =\left(1-\frac{V_{l}}{2 i \omega}\right) T_{I}^{r} .
\end{aligned}
$$


These satisfy the relations

$$
\begin{array}{r}
\left|R_{I}^{r}\right|^{2}+\left|T_{I}^{r}\right|^{2}=1, \\
\left|T_{I}^{l}\right|^{2}-\left|R_{I}^{l}\right|^{2}+\left|R_{I}^{r}\right|^{2}=1 .
\end{array}
$$

The negative sign in front of the $R_{I}^{l}$ term in (3.9b) comes from the fact that the reflected modes $R_{I}^{l} e^{-i \omega u}$ inside the $\mathrm{BH}$ have a negative norm [see Eq. (2.7)].

The asymptotic form of the in $f_{I}^{r}$ mode as $x \rightarrow+\infty$ is

$$
{ }^{\text {in }} f_{I}^{r}=\frac{e^{-i \omega t}}{\sqrt{4 \pi \omega}}\left[e^{-i \omega x^{*}}+R_{I}^{r} e^{i \omega x^{*}}\right]
$$

and

$$
{ }^{\operatorname{in}} f_{I}^{l}=\frac{e^{-i \omega t}}{\sqrt{4 \pi \omega}}\left[T_{I}^{l} e^{-i \omega x^{*}}+R_{I}^{l} e^{i \omega x^{*}}\right]
$$

for $x \rightarrow-\infty$.

Following the same procedure for the ${ }^{\text {in }} f_{H}^{r}$ modes coming out from the part of the past horizon in the $r$ region (see Fig. 8), we have

$$
\begin{aligned}
T_{H}^{r} & =\frac{1}{1-\frac{V_{r}}{2 i \omega}}, \\
R_{H}^{r} & =\frac{\frac{V_{r}}{2 i \omega}}{1-\frac{V_{r}}{2 i \omega}},
\end{aligned}
$$

satisfying $\left|R_{H}^{r}\right|^{2}+\left|T_{H}^{r}\right|^{2}=1$. Similarly, the ingoing $R_{H}^{r}$ part gets scattered by the $\delta$ function potential inside the horizon (as shown in Fig. 9) with the result

$$
\begin{gathered}
T_{H}^{l}=\left(1-\frac{V_{l}}{2 i \omega}\right) R_{H}^{r}, \\
R_{H}^{l}=\frac{V_{l}}{2 i \omega} R_{H}^{r}, \\
\stackrel{R_{H}^{r}}{\stackrel{\text { Incident }}{\longrightarrow}}
\end{gathered}
$$

FIG. 8. Illustration of a plane wave incident onto a potential from the left, which then is partially transmitted to the right and also partial reflected back to the left of the barrier. The reflected portion then travels into the interior of the $\mathrm{BH}$ where it encounters the potential in the interior. There the "reflected" and "transmitted" portions travel away from the potential to the left; see Fig. 9.

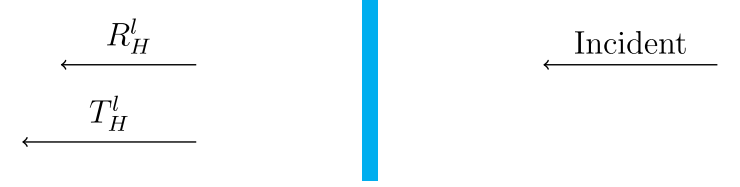

FIG. 9. Scattering inside the horizon of the mode in $f_{H}^{r}$.

again with $\left|T_{H}^{l}\right|^{2}-\left|R_{H}^{l}\right|^{2}+\left|T_{H}^{r}\right|^{2}=1$, leading to the asymptotic form

$$
{ }^{\text {in }} f_{H}^{r}=\frac{e^{-i \omega t}}{\sqrt{4 \pi \omega}} T_{H}^{r} e^{i \omega x^{*}}
$$

for $x \rightarrow+\infty$ and

$$
{ }^{\mathrm{in}} f_{H}^{r}=\frac{e^{-i \omega t}}{\sqrt{4 \pi \omega}}\left[R_{H}^{l} e^{-i \omega x^{*}}+T_{H}^{l} e^{i \omega x^{*}}\right]
$$

for $x \rightarrow-\infty$.

Finally, for the modes ${ }^{\text {in }} f_{H}^{l}$ coming from the part of the past horizon in region $l$ (see Fig. 10), the effective transmission and reflection coefficients are, respectively,

$$
\begin{aligned}
& \tilde{T}_{H}^{l}=1-\frac{V_{l}}{2 i \omega}, \\
& \tilde{R}_{H}^{l}=\frac{V_{l}}{2 i \omega},
\end{aligned}
$$

satisfying $\left|\tilde{T}_{H}^{l}\right|^{2}-\left|\tilde{R}_{H}^{l}\right|^{2}=1$. The asymptotic $(x \rightarrow-\infty)$ form of in $f_{H}^{l}$ is

$$
{ }^{\text {in }} f_{H}^{l}=\frac{e^{i \omega t}}{\sqrt{4 \pi \omega}}\left[\tilde{T}_{H}^{l} e^{-i \omega x^{*}}+\tilde{R}_{H}^{l} e^{i \omega x^{*}}\right] .
$$

Having defined the in basis, the field operator $\hat{\phi}^{(2)}$ can be expanded as

$\hat{\phi}^{(2)}=\int d \omega\left[{ }_{r}^{\mathrm{in}} \hat{a}_{I}\left({ }^{\mathrm{in}} f_{I}^{r}\right)+{ }_{r}^{\mathrm{in}} \hat{a}_{H}\left({ }^{\mathrm{in}} f_{H}^{r}\right)+{ }_{l}^{\mathrm{in}} \hat{a}_{H}\left({ }^{\mathrm{in}} f_{H}^{l}\right)+\right.$ H.c. $]$,

where the $\hat{a}$ 's are the annihilation operators for the respective modes.

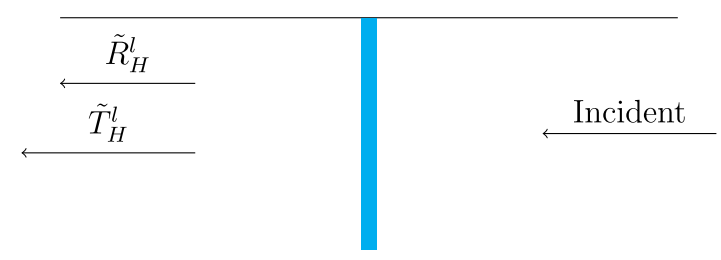

FIG. 10. Scattering of the mode in $f_{H}^{l}$. 


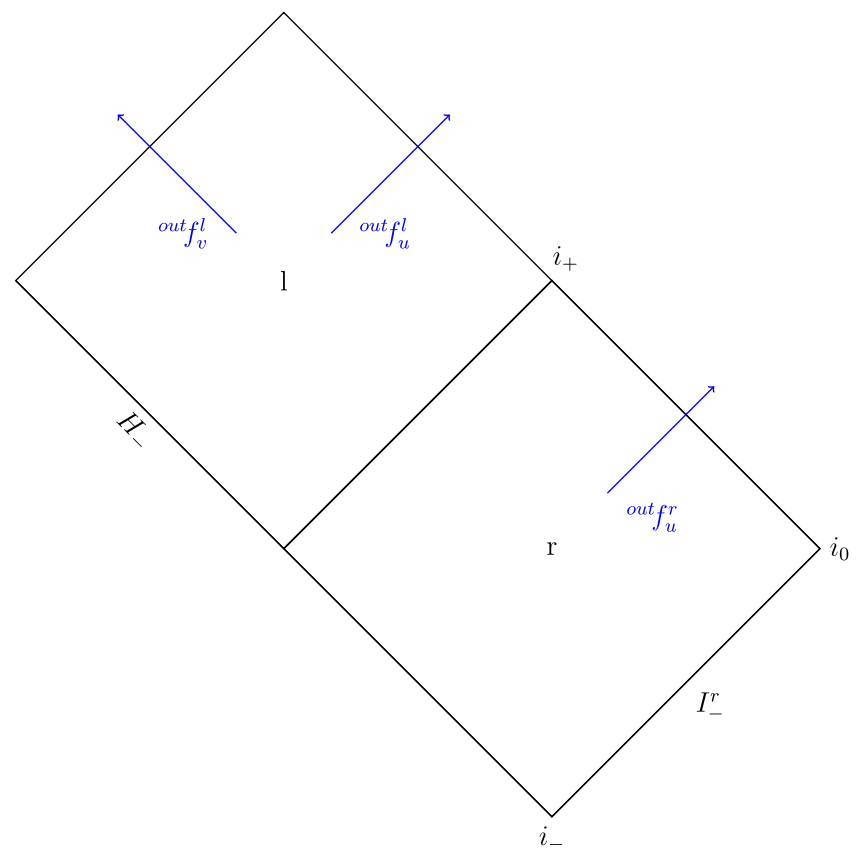

FIG. 11. Penrose diagram illustrating the modes forming the "out" basis.

Alternatively one can construct another basis called the out basis formed by modes having the asymptotic form

$$
{ }^{\text {out }} f_{u}^{r}=\frac{e^{-i \omega t}}{\sqrt{4 \pi \omega}} e^{i \omega x^{*}}=\frac{e^{-i \omega u}}{\sqrt{4 \pi \omega}}
$$

for $x \rightarrow+\infty$ and

$$
\begin{aligned}
& { }^{\text {out }} f_{u}^{l}=\frac{e^{i \omega t}}{\sqrt{4 \pi \omega}} e^{-i \omega x^{*}}=\frac{e^{i \omega u}}{\sqrt{4 \pi \omega}}, \\
& { }^{\text {out } f_{v}^{l}}=\frac{e^{-i \omega t}}{\sqrt{4 \pi \omega}} e^{-i \omega x^{*}}=\frac{e^{-i \omega v}}{\sqrt{4 \pi \omega}}
\end{aligned}
$$

for $x \rightarrow-\infty$. These modes are represented in the Penrose diagram in Fig. 11.

Proceeding in the same manner we can construct the ${ }^{\text {out } f}$ modes throughout the spacetime and then obtain the following expressions of the field operator:

$$
\begin{aligned}
\hat{\phi}^{(2)}= & \int d \omega\left[{ }_{r}^{\text {out }} \hat{a}_{u}\left({ }^{\text {out }} f_{u}^{r}\right)+{ }_{l}^{\text {out }} \hat{a}_{u}\left({ }^{\text {out }} f_{u}^{l}\right)\right. \\
& \left.+{ }_{r}^{\text {out }} \hat{a}_{v}\left({ }^{\text {out }} f_{v}^{r}\right)+\text { H.c. }\right],
\end{aligned}
$$

where the out $\hat{a}$ 's are annihilation operators. The in and out bases are related by a Bogoliubov transformation. Looking at the asymptotic form of the in modes Eqs. (3.10a), (3.10b), (3.13a), (3.13b) and (3.15), one can rewrite the modes on $I_{+}$as follows:

$$
\begin{aligned}
& { }^{\text {in }} f_{I}^{r}=R_{I}^{r \text { out }} f_{u}^{r}+T_{I}^{l \text { out }} f_{v}^{l}+R_{I}^{l \text { out }} f_{u}^{l *}, \\
& { }^{\text {in }} f_{H}^{r}=T_{H}^{r \text { out }} f_{u}^{r}+T_{H}^{l \text { out }} f_{v}^{l}+R_{H}^{l \text { out }} f_{u}^{l *}, \\
& { }^{\text {in }} f_{H}^{l}=\tilde{R}_{H}^{l \text { out }} f_{v}^{l *}+\tilde{T}_{H}^{l \text { out }} f_{u}^{l} .
\end{aligned}
$$

Note that there is no contribution to ${ }^{\text {in }} f_{H}^{l}$ from the ${ }^{\text {out }} f_{u}^{r}$ modes. Using the scattering $S$-matrix formalism we can write the relation between the two basis as

$$
\left(\begin{array}{c}
\text { in } f_{I}^{r} \\
{ }^{\mathrm{in}} f_{H}^{r} \\
{ }_{\mathrm{in}} f_{H}^{l^{*}}
\end{array}\right)=S^{T}\left(\begin{array}{c}
{ }^{\mathrm{out}} f_{u}^{r} \\
{ }^{\mathrm{out}} f_{v}^{l} \\
{ }_{\mathrm{out}} f_{u}^{l *}
\end{array}\right),
$$

where

$$
S^{T}=\left(\begin{array}{ccc}
S_{u_{r}, v_{r}} & S_{v_{l}, v_{r}} & S_{u_{l}, v_{r}} \\
S_{u_{r}, u_{r}} & S_{v_{l}, u_{r}} & S_{u_{l}, u_{r}} \\
0 & S_{v_{l}, u_{l}} & S_{u_{l}, u_{l}}
\end{array}\right)
$$

is the transpose of the scattering matrix $S$.

The notation used is borrowed from Ref. [13] and is quite intuitive. For example $S_{u_{r}, v_{r}}$ indicates an incoming $v$ mode from $r$ leading to an outgoing $u$ mode in $r$. The corresponding Bogoliubov transformation for the annihilation operators of the two bases is

$$
\left(\begin{array}{c}
{ }^{\text {out }} \hat{a}_{u}^{r} \\
\operatorname{out}_{\hat{a}}^{l} \\
\operatorname{out}_{\hat{a}}^{l+}{ }_{u}^{l \dagger}
\end{array}\right)=S\left(\begin{array}{c}
\text { in } \hat{a}_{I}^{r} \\
\text { in } \hat{a}_{H}^{r} \\
\text { in } \hat{a}_{H}^{l \dagger}
\end{array}\right),
$$

where

$$
S=\left(\begin{array}{ccc}
S_{u_{r}, v_{r}} & S_{u_{r}, u_{r}} & 0 \\
S_{v_{l}, v_{r}} & S_{v_{l}, u_{r}} & S_{v_{l}, u_{l}} \\
S_{u_{l}, v_{r}} & S_{u_{l}, u_{r}} & S_{u_{l}, u_{l}}
\end{array}\right) .
$$

For the two delta functions potential the $S$-matrix elements can be found by inspection of Eqs. (3.19a)-(3.19c) resulting in

$$
\begin{array}{ll}
S_{u_{r}, v_{r}}=R_{I}^{r}, & S_{u_{r}, u_{r}}=T_{H}^{r}, \\
S_{v_{l}, v_{r}}=T_{I}^{l}, & S_{v_{l}, u_{r}}=T_{H}^{l}, \\
S_{u_{l}, v_{r}}=R_{I}^{l}, & S_{u_{l}, u_{r}}=R_{H}^{l}, \\
S_{v_{l}, u_{l}}=\tilde{R}_{H}^{l *}, & S_{u_{l}, u_{l}}=\tilde{T}_{H}^{l *} .
\end{array}
$$

We are interested in the numbers of outgoing particles in the various channels, namely 


$$
\left\langle r_{r}^{\text {out }} \hat{a}_{u_{r}}^{\dagger \text { out }} \hat{a}_{u}\right\rangle, \quad\left\langle{ }_{l}^{\text {out }} \hat{a}_{v_{l}}^{\text {†out }} \hat{a}_{v}\right\rangle, \quad \text { and } \quad\left\langle{ }_{l}^{\text {out }} \hat{a}_{u_{l}}^{\dagger \text { out }} \hat{a}_{u}\right\rangle .
$$

To perform this calculation we have first to specify the quantum state of the $\hat{\phi}^{(2)}$ operator in which the expectation values in Eq. (3.23) have to be taken. The in modes used in the expression of the field operator $\hat{\phi}^{(2)}$ have a temporal part $e^{ \pm i \omega t}$. These are the eigenfunctions of the Killing vector $\frac{\partial}{\partial t}$ associated with the stationarity of the metric and are positive or negative (Killing) energy modes with respect to Schwarzschild time $t$. The quantum state associated with this expansion is annihilated by all the ${ }^{\text {in }} \hat{a}$ operators and is called the Boulware vacuum [14], i.e.,

$$
\begin{aligned}
\operatorname{in}_{I}^{r}|B\rangle & =0, \\
\operatorname{in}^{r} \hat{a}_{H}^{r}|B\rangle & =0, \\
{ }^{\operatorname{in}} \hat{a}_{H}^{l}|B\rangle & =0
\end{aligned}
$$

for all values of $\omega$. This is the most "natural" quantum state one can define on the extended manifold described by the Penrose diagram of Fig. 4. Physically $|B\rangle$ describes a state in which there are no incoming particles either from past right infinity $I_{-}^{r}$ or from the past horizon $H_{-}$. Although natural, this does not correctly describe the quantum state of the field $\hat{\phi}^{(2)}$ if the $\mathrm{BH}$ is formed by a dynamic gravitational collapse. The collapse in fact induces the conversion of quantum vacuum fluctuations to real on shell particles, the so-called Hawking radiation [7]. The state which correctly describes this process, at least at late times, is called the Unruh vacuum $|U\rangle$ [15]. The difference between the two states can be schematically summarized as follows. For the Unruh vacuum the modes coming out from the past horizon are chosen to be positive and negative frequency, not with respect to the Schwarzschild time $t$, but with respect to Kruskal time. Thus instead of the mode in $f_{u}^{r}$ and ${ }^{\text {in }} f_{u}^{l}$, the modes are chosen as

$$
\begin{gathered}
f_{H}^{K}=\frac{e^{-i \omega_{K} U}}{\sqrt{4 \pi \omega}}, \\
U=\mp \frac{e^{-\kappa u}}{\kappa},
\end{gathered}
$$

where the - and + refer to the $r$ and $l$ regions, respectively, and $\kappa$ is the surface gravity of the BH horizon, which for our metric is

$$
\kappa=\left.\frac{1}{2 c} \frac{d}{d x}\left(c^{2}-v^{2}\right)\right|_{x=0} .
$$

The modes coming from past null infinity for the Unruh vacuum are chosen as ${ }^{\mathrm{in}} f_{I}^{r}$. The field can then be expanded in terms of a complete set of these modes:

$$
\begin{aligned}
\hat{\phi}^{(2)}= & \int d \omega_{k}\left[\left(\hat{a}_{\omega_{K}} f_{H}^{K}+\hat{a}_{\omega_{K}}^{\dagger} f_{H}^{K *}\right)\right. \\
& +\int d \omega\left[{ }^{\mathrm{in}} \hat{a}_{I}^{r}\left(\mathrm{in}_{I}^{r}\right)+{ }^{\mathrm{in}} \hat{a}_{I}^{r \dagger}\left({ }^{\mathrm{in}} f_{I}^{r *}\right)\right] .
\end{aligned}
$$

The Unruh state is therefore defined as

$$
\begin{aligned}
& \hat{a}_{\omega_{K}}|U\rangle=0, \\
& { }^{\operatorname{in} \hat{a}_{I}^{r}|U\rangle}=0
\end{aligned}
$$

for every $\omega$ and $\omega_{K}$. The relation between the two sets of operators is given by the following Bogoliubov transformations:

$$
\begin{aligned}
& \operatorname{in}_{\hat{a}_{H}^{r}}^{r}=\int d \omega_{k}\left[\alpha_{\omega_{K} \omega}^{r} \hat{a}_{\omega_{K}}+\beta_{\omega_{K} \omega}^{r *} \hat{a}_{\omega_{K}}^{\dagger}\right], \\
& \operatorname{in} \hat{a}_{H}^{l}=\int d \omega_{k}\left[\alpha_{\omega_{K} \omega}^{l} \hat{a}_{\omega_{K}}+\beta_{\omega_{K} \omega}^{l *} \hat{a}_{\omega_{K}}^{\dagger}\right],
\end{aligned}
$$

where the Bogoliubov coefficients are given by (see e.g., [16])

$$
\begin{aligned}
& \alpha_{\omega_{K} \omega}^{r}=\frac{1}{2 \pi \kappa} \sqrt{\frac{\omega}{\omega_{K}}}\left(-i \omega_{K}\right)^{i \omega / \kappa} \Gamma\left(\frac{-i \omega}{\kappa}\right), \\
& \beta_{\omega_{K} \omega}^{r}=\frac{1}{2 \pi \kappa} \sqrt{\frac{\omega}{\omega_{K}}}\left(-i \omega_{K}\right)^{-(i \omega / \kappa)} \Gamma\left(\frac{i \omega}{\kappa}\right), \\
& \alpha_{\omega_{K} \omega}^{l}=\frac{1}{2 \pi \kappa} \sqrt{\frac{\omega}{\omega_{K}}}\left(i \omega_{K}\right)^{-(i \omega / \kappa)} \Gamma\left(\frac{i \omega}{\kappa}\right), \\
& \beta_{\omega_{K} \omega}^{l}=\frac{1}{2 \pi \kappa} \sqrt{\frac{\omega}{\omega_{K}}}\left(i \omega_{K}\right)^{i \omega / \kappa} \Gamma\left(\frac{-i \omega}{\kappa}\right) .
\end{aligned}
$$

Using the Bogoliubov transformations, Eqs. (3.21a) and (3.29), we obtain

$$
\begin{aligned}
& n_{u}^{r} \equiv\left\langle\left. U\right|_{r} ^{\text {out }} \hat{a}_{u_{r}}^{\dagger \text { out }} \hat{a}_{u} \mid U\right\rangle=\int d \omega_{K}\left|S_{u_{r}, u_{r}}\right|^{2}\left|\beta_{\omega_{K} \omega}^{r}\right|^{2}, \\
& n_{v}^{l} \equiv\left\langle\left. U\right|_{l} ^{\text {out }} \hat{a}_{v l}^{\dagger \text { out }} \hat{a}_{v} \mid U\right\rangle \\
& =\int d \omega_{K}\left[\left|S_{v_{l}, u_{r}}\right|^{2}\left|\beta_{\omega_{K} \omega}^{r}\right|^{2}+S_{v_{l}, u_{r}}^{*} \beta_{\omega_{K} \omega}^{r} S_{v_{l}, u_{l}}^{*} \alpha_{\omega_{K} \omega}^{l *}\right. \\
& \left.+S_{v_{l}, u_{l}} \alpha_{\omega_{K} \omega}^{l} S_{v_{l}, u_{r}} \beta_{\omega_{K} \omega}^{r *}+\left|S_{v_{l}, u_{l}}\right|^{2}\left|\alpha_{\omega_{K} \omega}^{l}\right|^{2}\right], \\
& n_{u}^{l} \equiv\left\langle\left. U\right|_{l} ^{\text {out }} \hat{a}_{u_{l}}^{\text {out }} \hat{a}_{u} \mid U\right\rangle \\
& =\int d \omega_{K}\left[\left|S_{u_{l}, u_{r}}\right|^{2}\left|\alpha_{\omega_{K} \omega}^{r}\right|^{2}+S_{u_{l}, u_{r}}^{*} \alpha_{\omega_{K} \omega}^{r *} S_{u_{l}, u_{l}} \beta_{\omega_{K} \omega}^{l}\right. \\
& \left.+S_{u_{l}, u_{r}} \alpha_{\omega_{K} \omega}^{r} S_{u_{l}, u_{l}}^{*} \beta_{\omega_{K} \omega}^{l *}+\left|S_{u_{l}, u_{l}}\right|\left|\beta_{\omega_{K} \omega}^{l}\right|^{2}\right]+\left|S_{u_{l}, v_{r}}\right|^{2} .
\end{aligned}
$$

One can see the combined effect of the near horizon mixing (the $\alpha$ and $\beta$ ) encoded in the Bogoliubov transformation 


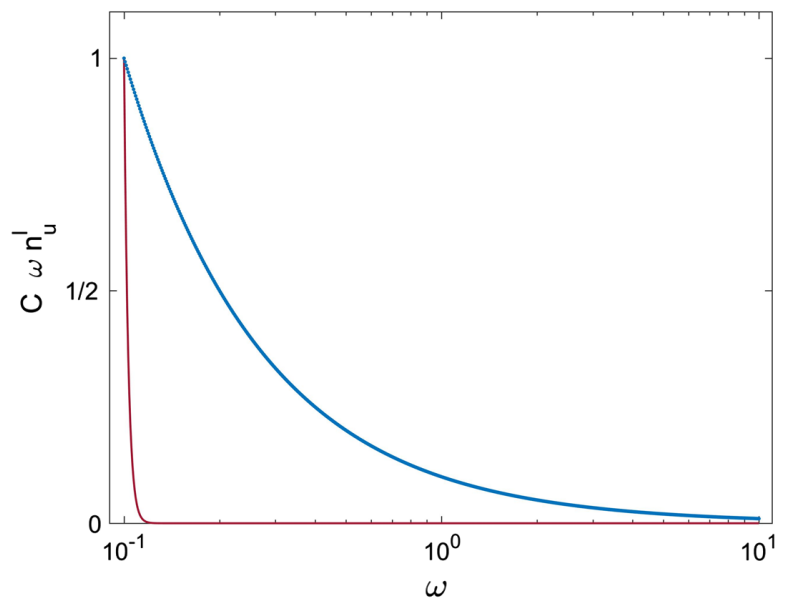

FIG. 12. Plots for $\omega \gg \kappa$ with $-V_{l}=V_{r} / 10=\kappa / 100$ (blue curve, upper) and $V_{l}=V_{r}=0$ (red curve, lower). The quantity $C$ $\omega n_{u}^{l}$, where $C$ is a scaling factor whose value is chosen for each curve so that $C \omega n_{u}^{l}=1$ for $\omega=10^{-1}$.

(3.29), which engenders Hawking thermal radiation, and the scattering caused by the potential (the $S$-matrix element). After some calculations we obtain

$$
\begin{aligned}
n_{u}^{r}= & \frac{4 \omega^{2}}{4 \omega^{2}+V_{R}^{2}} \frac{1}{e^{2 \pi \omega / \kappa}-1}, \\
n_{v}^{l}= & \frac{1}{4 \omega^{2}}\left|V_{r} \frac{2 i \omega-V_{l}}{2 i \omega-V_{r}}-V_{l} e^{\pi \omega / \kappa}\right|^{2} \frac{1}{e^{2 \pi \omega / \kappa}-1}, \\
n_{u}^{l}= & \frac{1}{4 \omega^{2}}\left|e^{\pi \omega / \kappa} \frac{V_{r} V_{l}}{2 i \omega+V_{r}}+\left(2 i \omega-V_{l}\right)\right|^{2} \\
& \times \frac{1}{e^{2 \pi \omega / \kappa}-1}+\frac{V_{l}^{2}}{4 \omega^{2}+V_{r}^{2}} .
\end{aligned}
$$

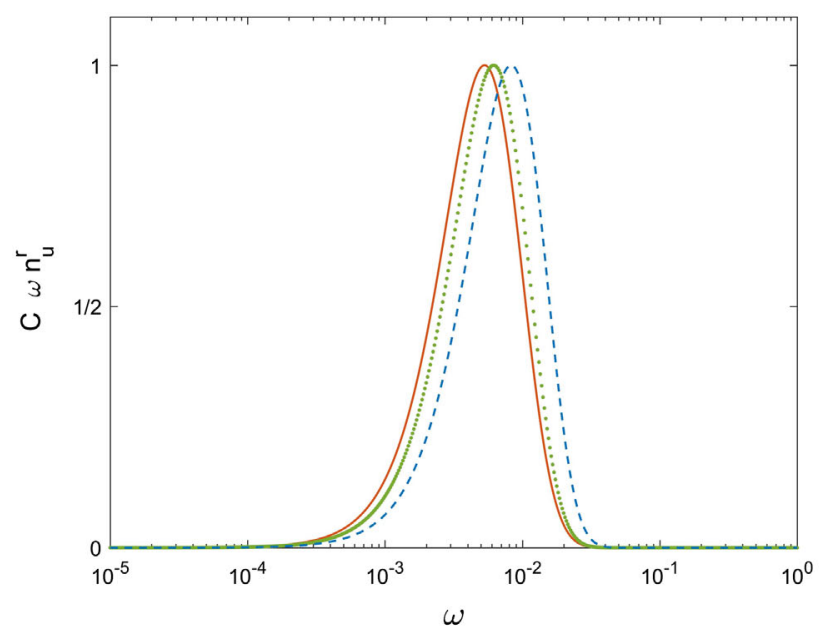

Note that we have omitted factors of $\delta(0)$ that arise from the normalization of plane waves. If wave packets are used, then such factors can be set equal to 1 .

One can verify that $n_{u}^{r}+n_{v}^{l}=n_{u}^{l}$. Thus the number of positive energy excitations created equals the number of negative ones as energy conservation requires.

One notices immediately the striking difference between the emission in the exterior region compared to that of the interior region. In the exterior region the scattering is the standard one; $n_{u}^{r}$ describes, as expected, a thermal emission at the temperature $T_{H}=\frac{\hbar \kappa}{2 \pi k_{B}}$ modulated by the graybody factor $\frac{4 \omega^{2}}{4 \omega^{2}+V_{r}^{2}}$ which regulates the infrared divergence associated with the Planckian distribution. The graybody factor goes to one for $\omega \gg V_{r}$. In the interior region the scattering is anomalous resulting in additional particle production. The distribution of the particles is infrared divergent-i.e, the spectrum is dominated by soft phonons - and it is not thermal. For the model we consider both $n_{u}^{l}$ and $n_{v}^{l}$ decay as a power law.

The high-frequency behavior of $n_{u}^{l}$ is shown in Fig. 12 for the cases $-V_{l}=V_{r} / 10$ and $V_{l}=V_{r}=0$. The more rapid, exponential, falloff in the latter case is clearly apparent. The low-frequency behaviors of $n_{u}^{r}$ and $n_{u}^{l}$ for various values of $V_{r}>0$ and $V_{l}<0$ are shown in Fig. 13. For both low and high frequencies the qualitative behaviors of $n_{v}^{\ell}$ for the same cases are identical to those of $n_{u}^{l}$. In addition to the infrared divergence mentioned above, the plots of in $n_{u}^{l}$ in Fig. 13 show another nontrivial feature, a peak, that arises in the quantity $\omega n_{u}^{l}$. (It also occurs for $\omega n_{v}^{l}$.) The peak appears to be most pronounced when $\left|V_{l}\right| \gg V_{r} \sim \kappa /(2 \pi)$. In this regime, the position, in $\omega$, of the peak is proportional to $V_{r}$ so it moves to the right on a plot of $\omega n_{u}^{l}$ versus $\omega$ as $V_{r}$ increases. For $V_{r} \gg \kappa /(2 \pi)$ it

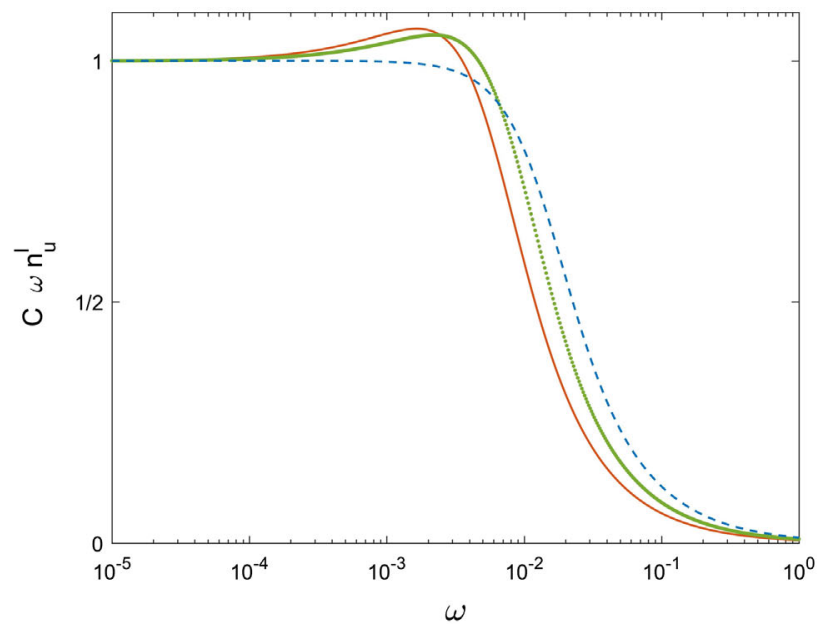

FIG. 13. Plots are shown of $\omega n_{u}^{r}$ and $\omega n_{u}^{l}$ for $-V_{l}=V_{r} / 10=\kappa / 100$ (blue, dashed curve), $-V_{l}=V_{r} / 100=\kappa / 100$ (green, dotted curve) and $-V_{l}=3 V_{r} / 200=\kappa / 100$ (orange, solid curve). Left: $C$ is a scaling factor whose value is chosen for each curve so that $C \omega$ $n_{u}^{r}=1$ at the peak of that curve. Right: $C$ is a scaling parameter whose value is chosen for each curve so that $C \omega n_{u}^{l}=1$ at $\omega=10^{-5}$. An unexpected peak in $C \omega n_{u}^{l}$ exists in all three cases but is only clearly visible in two. 
disappears, because it becomes lost in the power law decay that occurs at high frequencies. In contrast, as $V_{r}$ gets smaller and moves to the left on the plot, the height of the peak decreases relative to its base. For small values of $\omega$ the height of the base is given by the value of $\omega n_{u}^{l}$ in the limit that $\omega \rightarrow 0$.

If, for fixed $V_{r},\left|V_{l}\right|$ decreases but is still larger than $V_{r}$, then the height of the peak also decreases. However, its location stays about the same. When $V_{r}=\left|V_{l}\right|$ the peak no longer exists. This can be shown analytically by looking at the derivative of $\omega n_{v}^{l}$ :

$$
\begin{aligned}
\frac{d\left(\omega n_{v}^{l}\right)}{d \omega} & =\frac{V_{r}^{2}\left(\kappa\left(1-e^{2 \pi \omega / \kappa}\right)+2 \pi \omega e^{\pi \omega / \kappa}\right)}{4 \kappa \omega^{2}\left(e^{\pi \omega / \kappa}+1\right)^{2}} \\
& =\frac{V_{r}^{2} \kappa e^{\pi \omega / 2 \kappa}\left(\frac{\pi \omega}{\kappa}-\sinh \left(\frac{\pi \omega}{\kappa}\right)\right)}{2 \kappa \omega^{2}\left(e^{\pi \omega / \kappa}+1\right)^{2}},
\end{aligned}
$$

which is less than zero for all $\omega>0$. Thus there is no peak like the one seen in Fig. 13 in the $V_{r}<\left|V_{l}\right|$ case. The same can also be shown for $n_{u}^{\ell}$, but the expressions are more complicated.

This peak is also present in other, more realistic, configurations for the effective potential. This will be shown elsewhere.

We make a final remark concerning the Boulware vacuum $|B\rangle$. This state is characterized by being a vacuum state at infinity $I_{ \pm}^{r}$ (no incoming and no outgoing particles for $x \rightarrow+\infty$ ), that is singular at $H_{ \pm}$. Indeed the number of particles created in the $r$ region is

$$
N_{u}^{r} \equiv\left\langle\left. B\right|_{r} ^{\text {out }} \hat{a}_{u r}^{\dagger \text { out }} \hat{a}_{u} \mid B\right\rangle=0
$$

This is not true in the $\mathrm{BH}$ interior region because of the particle production that occurs there resulting in

$N_{v}^{l} \equiv\left\langle B\left|{ }_{l}^{\text {out }} \hat{a}_{v l}^{\dagger \text { out }} \hat{a}_{v}\right| B\right\rangle=\left|S_{v_{l}, u_{l}}\right|^{2}=\left|\tilde{R}_{H}^{l *}\right|^{2}=\frac{V_{l}^{2}}{4 \omega^{2}}$

and

$$
\begin{aligned}
N_{u}^{l} & \equiv\left\langle\left. B\right|_{l} ^{\text {out }} \hat{a}_{u l}^{\text {†out }} \hat{a}_{u} \mid B\right\rangle=\left|S_{u_{l}, v_{l}}\right|^{2}+\left|S_{u_{l}, v_{l}}\right|^{2} \\
& =\left|R_{I}^{l}\right|^{2}+\left|R_{H}^{l}\right|^{2}=\frac{V_{l}^{2}}{4 \omega^{2}}\left[\left|R_{H}^{r}\right|^{2}+\left|T_{H}^{r}\right|^{2}\right]=\frac{V_{l}^{2}}{4 \omega^{2}} .
\end{aligned}
$$

Inside the $\mathrm{BH},|B\rangle$ is no longer an out vacuum state. Instead there is a net flux of particles (of positive and negative energy) directed toward $x \rightarrow-\infty$ with $N_{v}^{l}=N_{u}^{l}$.

\section{THE MASSIVE MODEL}

The second toy model we want to investigate is the one introduced in Ref. [12], where in the field equation (2.4) $V_{\text {eff }}$ is neglected and the mass term $k_{\perp}^{2}\left(c^{2}-v_{0}^{2}\right)$ is approximated as two step functions (see Fig. 14):

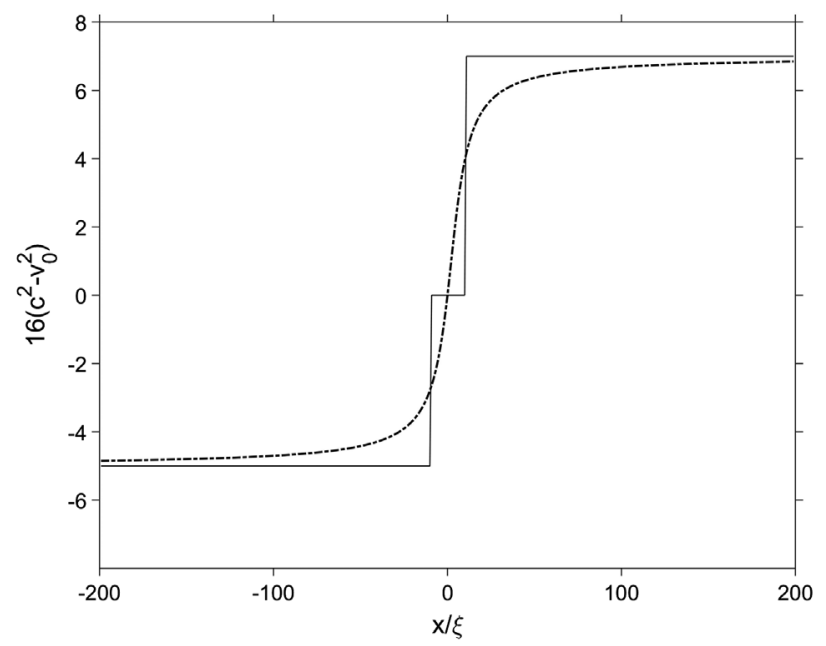

FIG. 14. Plot from Ref. [12] of the coefficient of $m^{2}$ (dotdashed curve) and the approximation to that coefficient (solid curve). This result is based on the sound speed profile used in Refs. [11,16].

$$
k_{\perp}^{2}\left(c^{2}-v_{0}^{2}\right) \rightarrow \begin{cases}m_{r}^{2} \Theta\left(x^{*}-x_{0 r}^{*}\right), & x>0, \\ -m_{l}^{2} \Theta\left(x^{*}-x_{0 l}^{*}\right), & x<0,\end{cases}
$$

with $m_{r}^{2}=m^{2}\left(c_{r}^{2}-v_{0}^{2}\right)$ and $m_{l}^{2}=m^{2}\left(v_{0}^{2}-c_{l}^{2}\right)$. Again $c_{r}$ and $c_{l}$ are the asymptotic values of $c(x)$ as $x \rightarrow+\infty$ and $x \rightarrow-\infty$, respectively. The - sign in front of $m_{l}^{2}$ comes from the fact that, inside the $\mathrm{BH}, c^{2}<v_{0}^{2}$. We also choose $x_{0 l}^{*}=0=x_{0 r}^{*}$ for simplicity. The field equation (2.4) simplifies to

$$
\begin{array}{ll}
{\left[-\partial_{t}^{2}+\partial_{x^{*}}^{2}-m_{r}^{2} \Theta\left(x^{*}\right)\right] \hat{\phi}^{(2)}=0,} & x>0, \\
{\left[-\partial_{t}^{2}+\partial_{x^{*}}^{2}+m_{l}^{2} \Theta\left(x^{*}\right)\right] \hat{\phi}^{(2)}=0,} & x<0 .
\end{array}
$$

Since the construction of the in basis for this model has been performed in Ref. [12], here we briefly sketch the basic features. The asymptotic form of the incoming $v$ modes that originate at $x=+\infty$ is

$$
{ }^{\text {in }} f_{I}^{r}=\frac{1}{\sqrt{4 \pi \omega}} e^{-i \omega t} e^{-i k_{r} x^{*}}
$$

with $k_{r} \equiv \sqrt{\omega^{2}-m_{r}^{2}}$. These are massive modes and they exist only if $\omega>m_{r}$; i.e., there is, as usual, a mass gap.

On $H^{-}$the modes are massless so

$$
\begin{aligned}
& { }^{\text {in }} f_{H}^{r}=\frac{1}{\sqrt{4 \pi \omega}} e^{-i \omega u}, \\
& { }^{\text {in }} f_{H}^{l}=\frac{1}{\sqrt{4 \pi \omega}} e^{i \omega u} .
\end{aligned}
$$

The forms of these modes throughout the spacetime can be found by enforcing continuity of the spatial part $\chi$ of the 


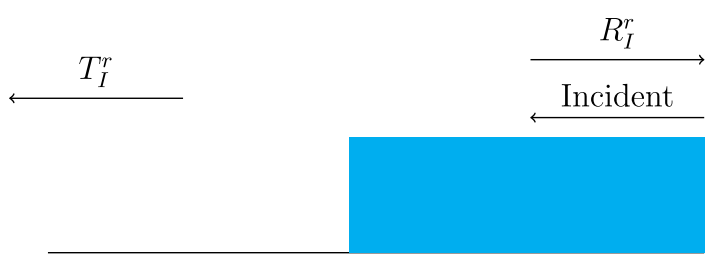

FIG. 15. Illustration of a plane wave incident from $x=+\infty$, which then is partially transmitted and partial reflected back.

modes and their derivatives at the boundaries of the step functions with the result

${ }^{\text {in }} f_{I}^{r}=\frac{e^{-i \omega t}}{\sqrt{4 \pi \omega}}\left[\frac{k_{r}-\omega}{k_{r}+\omega} e^{i k_{r} x^{*}}+e^{-i k_{r} x^{*}}\right], \quad$ for $x \rightarrow+\infty$,

${ }^{i n} f_{I}^{r}=\frac{e^{-i \omega t}}{\sqrt{4 \pi k_{l}}} \frac{\sqrt{k_{l} k_{r}}}{k_{r}+\omega}\left[\frac{k_{l}-\omega}{2 k_{l}} e^{i k_{l} x^{*}}+\frac{k_{l}+\omega}{2 k_{l}} e^{-i k_{l} *^{*}}\right]$,

for $x \rightarrow-\infty$,

where $k_{l} \equiv \sqrt{\omega^{2}+m_{l}^{2}}$. Note that, unlike $k_{r}, k_{l}$ is real for any value of $\omega$ and $k_{l} \geq m_{l}$. These modes can be illustrated schematically in the same way as the previous toy model of Sec. III. The scattering of these modes in the exterior is illustrated in Fig. 15, while Fig. 16 illustrates the interior scattering.

The $R_{I}^{r}$ part is the coefficient of the first exponential in Eq. (4.5a), while $T_{I}^{l}$ and $R_{I}^{l}$ are the coefficients of the first and second exponentials, respectively, in Eq. (4.5b).

For the ${ }^{\text {in }} f_{H}^{r}$ modes one finds

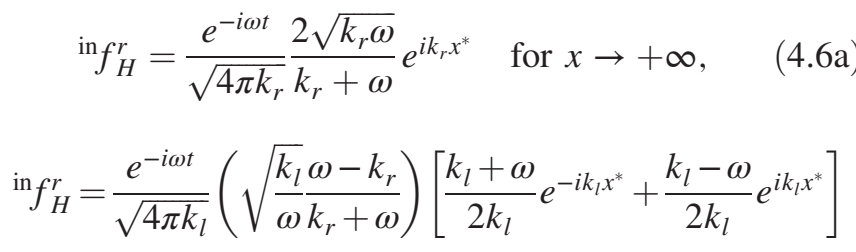

$$
\text { for } x \rightarrow-\infty \text {. }
$$

Schematically the exterior scattering is described in Fig. 17, and the inner one is similar to the one represented in Fig. 16.

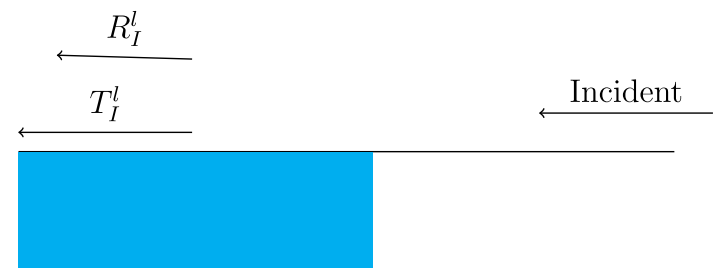

FIG. 16. Illustration of a plane wave incident onto the negative step function potential from the right. As this is in the interior of the $\mathrm{BH}$ both the transmitted and reflected portions of the mode are forced to travel further into the $\mathrm{BH}$.
The $T_{H}^{r}$ term is the coefficient of the first exponential in Eq. (4.6a), while $T_{H}^{l}$ and $R_{H}^{l}$ are the coefficients of the first and second exponential, respectively, in Eq. (4.6b). Note that for $\omega<m_{r}$ the ${ }^{\text {in }} f_{H}^{r}$ mode coming from $H_{-}^{r}$ is completely reflected at $x_{0 r}^{*}$. This is the boomerang effect as seen in Ref. [17].

The final set of modes in this basis are the in $f_{H}^{l}$ modes which are

${ }^{\text {in }} f_{H}^{l}=\frac{e^{i \omega t}}{\sqrt{4 \pi k_{l}}} \sqrt{\frac{k_{l}}{\omega}}\left[\frac{k_{l}+\omega}{2 k_{l}} e^{-i k_{l} x^{*}}+\frac{k_{l}-\omega}{2 k_{l}} e^{i k_{l} x^{*}}\right]$ for $x \rightarrow-\infty$.

Schematically this is the same as seen in Fig. 16. $\tilde{T}_{H}^{l}$ is the coefficient of the first exponential in Eq. (4.7), and $\tilde{R}_{H}^{l}$ is the coefficient of the second one.

The out basis is constructed by using a similar procedure to that described in the previous section. Starting from the asymptotic forms of the modes

$$
{ }^{\mathrm{out}} f_{u}^{r}=\frac{1}{\sqrt{4 \pi k_{r}}} e^{-i \omega t} e^{i k_{r} x^{*}}
$$

as $x \rightarrow+\infty$, and

$$
\begin{gathered}
{ }^{\text {out }} f_{u}^{l}=\frac{1}{\sqrt{4 \pi k_{l}}} e^{i \omega t} e^{-i k_{l} x^{*}}, \\
{ }^{\text {out }} f_{v}^{l}=\frac{1}{\sqrt{4 \pi k_{l}}} e^{-i \omega t} e^{-i k_{l} x^{*}},
\end{gathered}
$$

for $x \rightarrow-\infty$. Using Eqs. (4.5a)-(4.7) we can express the in modes in terms of the out modes as

in $f_{I}^{r}=\frac{k_{r}-\omega}{k_{r}+\omega}{ }_{\text {out }} f_{u}^{r}+\frac{2 \sqrt{k_{l} k_{r}}}{k_{r}+\omega}\left[\frac{k_{l}+\omega}{2 k_{l}}{ }^{\text {out }} f_{v}^{l}+\frac{k_{l}-\omega}{2 k_{l}}{ }^{\text {out }} f_{u}^{l *}\right]$,

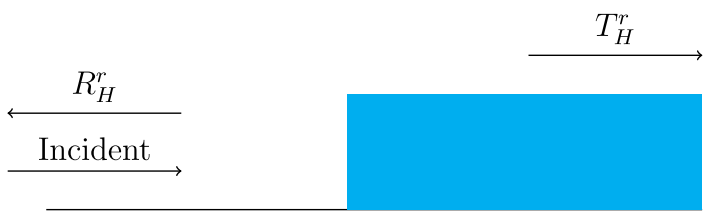

FIG. 17. Illustration of a plane wave incident onto a step function potential from the left, which then is partially transmitted to the right and also partial reflected back to the left of the barrier. There, the reflected portion then travels into the interior of the $\mathrm{BH}$ where it encounters the step function potential where the anomalous scattering occurs. The reflected and transmitted portions travel away from the potential to the left; see Fig. 16. 

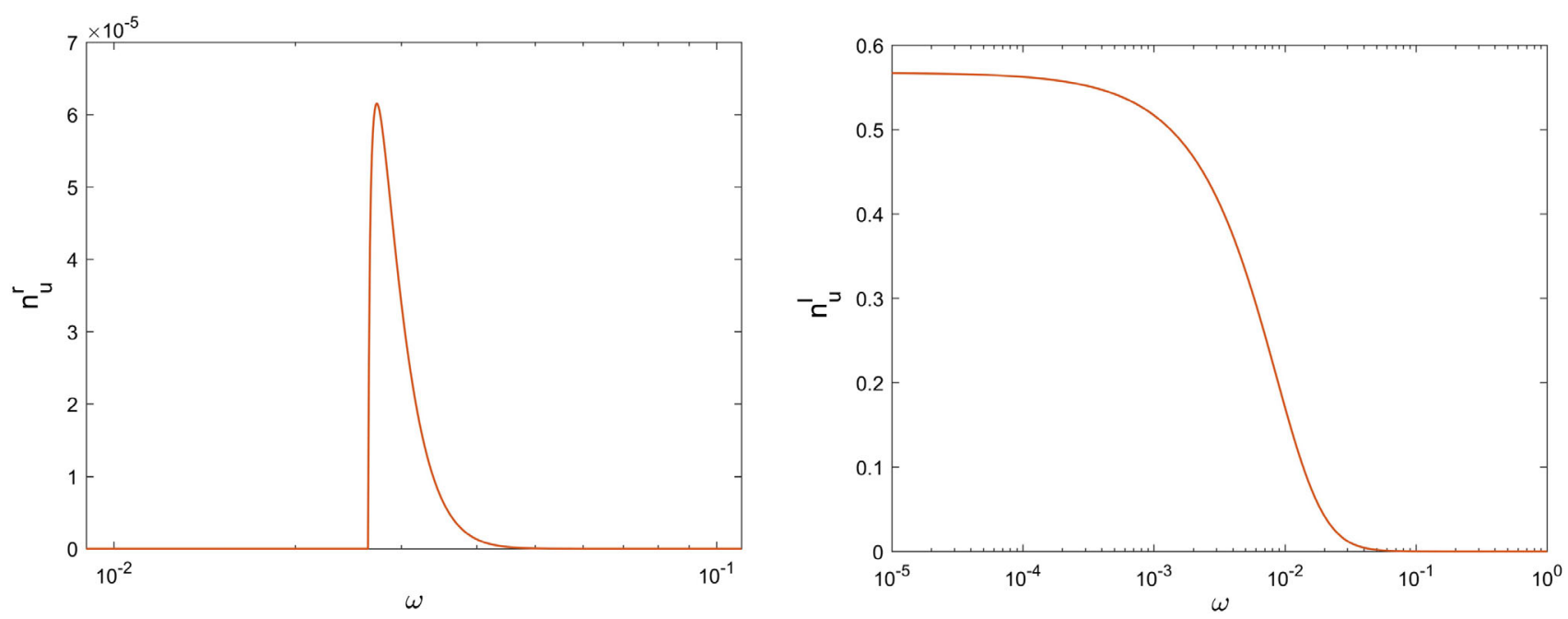

FIG. 18. Plots for $m=4 \times 10^{-2}$. Left: Plot of $n_{u}^{r}$ vs $\omega$. Note that, for $\omega<m_{r}, n_{u}^{r}=0$ because no modes reach future null infinity in the $r$ region. Thus there is a discontinuity in $n_{u}^{r}$ at $\omega=m_{r}$. Right: Plot of $n_{u}^{l}$ vs $\omega$ which shows the nonthermal nature of the interior particle number, as the low $\omega$ behavior is shown to approach a constant and for large $\omega$ it decays as a power law. The qualitative behavior of $n_{v}^{l}$ is very similar to $n_{u}^{l}$; thus it is not shown.

$$
\begin{aligned}
\operatorname{in}_{H}^{r}= & \frac{2 \sqrt{k_{r} \omega}}{k_{r}+\omega} \text { out } f_{u}^{r} \\
& +\left(\sqrt{\frac{k_{l}}{\omega}} \frac{\omega-k_{r}}{k_{r}+\omega}\right)\left[\frac{k_{l}+\omega}{2 k_{l}} \text { out } f_{v}^{l}+\frac{k_{l}-\omega}{2 k_{l}} \text { out } f_{u}^{l *}\right] \\
& \operatorname{in}_{H}^{l}=\sqrt{\frac{k_{l}}{\omega}}\left[\frac{k_{l}-\omega}{2 k_{l}} \text { out }_{v}^{l *}+\frac{k_{l}+\omega}{2 k_{l}} \text { out } f_{u}^{l}\right]
\end{aligned}
$$

Note that there is no contribution to in $f_{H}^{l}=0$ from the out $f_{u}^{r}$ modes. From these the Bogoliubov transformations between the in and out creation and annihilation operators can be found as in the previous section. After some calculations we obtain the following expressions for the number of outgoing created particles in the Unruh state:

$$
\begin{gathered}
n_{u}^{r}=\frac{4 k_{r} \omega}{\left(\omega+k_{r}\right)^{2}} \frac{1}{e^{2 \pi \omega / \kappa}-1} \Theta\left(\omega-m_{r}\right), \\
n_{v}^{l}=\frac{1}{4 k_{l} \omega}\left|\left(k_{l}+\omega\right) \frac{\omega-k_{r}}{\omega+k_{r}}+\left(k_{l}-\omega\right) e^{\pi \omega / \kappa}\right|^{2} \frac{1}{e^{2 \pi \omega / \kappa}-1}, \\
n_{u}^{l}=\frac{1}{4 k_{l} \omega}\left|e^{\pi \omega / \kappa}\left(k_{l}-\omega\right) \frac{\omega-k_{r}}{\omega+k_{r}}+\left(k_{l}+\omega\right)\right|^{2} \\
\times \frac{1}{e^{2 \pi \omega / \kappa}-1}+\frac{k_{r}}{k_{l}}\left(\frac{k_{l}-\omega}{k_{l}+\omega}\right)^{2} \Theta\left(\omega-m_{r}\right) .
\end{gathered}
$$

Note that we have omitted factors of $\delta(0)$ that arise from the normalization of plane waves. If wave packets are used, then such factors can be set equal to 1 . One can verify that $n_{u}^{r}+n_{v}^{l}=n_{u}^{l}$ above the threshold $\omega>m_{r}$, while for $0<$ $\omega<m_{r}$ we have $n_{v}^{l}=n_{u}^{l}$.

We find that, unlike the exterior region, the emission inside is not thermal. Furthermore, $n_{u}^{l}$ and $n_{v}^{l}$ are finite in the infrared $\omega \rightarrow 0$ limit (see also Ref. [18]). In the asymptotic interior region $x \rightarrow-\infty$, the dispersion relation for the massive modes is $\omega^{2}-k^{2}=-m_{l}^{2}$ so there is no threshold for the conserved energy, and one can have phonons whose energy is below $m_{l}$; even a zero frequency mode with $|k|=m_{l}$ exists. There is a threshold in momentum $|k|>m_{l}$ for the outgoing $x \rightarrow-\infty$ particles. These features are a consequence of the switching roles between $t$ and $x^{*}$ inside the $\mathrm{BH}$ as we have discussed previously. Note, however, that, unlike the energy, the momentum is not conserved along the trajectory of the created particle. Finally, the energy of the $(u, l)$ particles is negative. All of these unusual features exist only inside the $\mathrm{BH}$. The deviation from a thermal spectrum is easily seen in Fig. 18. Note that the spectrum in the exterior is truncated for modes where $\omega<m_{R}$.

For completeness we can also work out the numbers of created particles in the Boulware state $|B\rangle$ :

$$
\begin{gathered}
N_{u}^{r}=0, \\
N_{u}^{l}=N_{v}^{l}=\frac{\left(k_{l}-\omega\right)^{2}}{4 \omega k_{l}} .
\end{gathered}
$$

One can see that, just as in the massless case, $|B\rangle$ is no longer an out vacuum state in the interior of the $\mathrm{BH}$. Moreover, unlike what happens in the Unruh state, the number of created particles diverges as $\omega \rightarrow 0$. 


\section{CONCLUSIONS}

We have investigated scattering in the exterior of the acoustic horizon of a $\mathrm{BEC}$ analog $\mathrm{BH}$ and anomalous scattering or particle production in its interior in a simple model with massless phonons and a different one for massive phonons. We have considered both the Unruh and Boulware states. The latter is the natural vacuum state for a static star, while the former gives a good approximation in the exterior region to the late time radiation produced by the black hole. As expected we find for the region outside the horizon that the spectrum at infinity is thermal modulo the graybody factor for the Unruh state and there are no particles for the Boulware state. In the massive case we find that, as expected, the emitted thermal radiation in the exterior is gapped.

In the interior anomalous scattering produces additional particle production for both massless and massive phonons and this destroys the thermal nature of the spectrum for the Unruh state. At small frequencies the emission is dominated by soft phonons but only in the massless case. At high frequencies one finds that, for the models considered, the particle number falls off like an inverse power of the frequency rather than exponentially. Not surprisingly particle production also occurs for the Boulware state in the interior. Thus the Boulware state remains a vacuum state in the exterior but can only be considered to be an initial vacuum state in the interior.

For massless phonons an unexpected peak was found in the quantities $\omega n_{u}^{\ell}$ and $\omega n_{v}^{\ell}$ when they are plotted as functions of $\omega$, with $n_{u}^{\ell}$ and $n_{v}^{\ell}$ the number of right-moving and left-moving particles found at future null infinity in the interior. This peak represents a clear deviation from a thermal spectrum. It occurs for a limited range of the factors $V_{r}$ and $V_{\ell}$ in the two delta function potential (3.1).

The presence of particle creation even for the Boulware state inside a $\mathrm{BH}$ can be understood by the fact that the Killing vector $\frac{\partial}{\partial t}$, of which the Boulware modes in are eigenfunctions, is spacelike inside the horizon. The symmetry associated with it is homogeneity rather than stationarity. This is clearly seen by the switch in these roles of the coordinates $t$ and $x^{*}$ inside the $\mathrm{BH} ; x^{*}$ is timelike and $t$ is spacelike, so a potential depending on $x^{*}$ is a time-dependent potential which, as such, causes particle creation.

Particle production associated with anomalous scattering induced by curvature and consequent deviation from a thermal distribution of the Hawking radiation was first noticed by Corley and Jacobson [19] in a different context in the region exterior to the event horizon. Specifically, they introduced an ad hoc modification of the two-dimensional wave equation for the modes propagating in a $\mathrm{BH}$ metric which results in a nonlinear dispersion relation, subluminal in their case, i.e., $\omega-v k= \pm \sqrt{k^{2}-\frac{k^{4}}{k_{0}^{2}}}$. Then they analyzed the influence of the induced anomalous scattering on the spectrum of the particles radiated by the $\mathrm{BH}$ in the region exterior to the horizon. The fact that the anomalous scattering occurs outside the horizon is a peculiar effect of the dispersion relation they chose. In a genuine general relativity framework, like the one we use, anomalous scattering and related particle production can occur only inside the horizon; outside the horizon, scattering is always the standard one giving just a graybody factor with no extra particle production.

Deviation of the Hawking radiation from a thermal distribution in the context of BEC analog BHs, where the modification of the relativistic dispersion relation is superluminal, i.e., $\omega-v k= \pm \sqrt{k^{2}+\frac{k^{4}}{k_{0}^{2}}}$, was first analyzed numerically by Macher and Parentani [5].

Our results are in the context of quantum field theory in curved space; as such, they involve a strictly linear dispersion relation for which there are no superluminal or subluminal modes. The connection to actual analog BHs is that our results should be valid for long-wavelength phonons for which the mode equation is approximately the same as that for a massless minimally coupled scalar field in the analog spacetime [16]. The connection of our results to real black holes is that, in the interior (where the Killing vector is spacelike), the spacetime is dynamic and there is also an effective potential, this time due to the spacetime curvature, and so nonthermal particle production should also occur.

The advantage of analog gravity is that, unlike what happens in the gravitational context, one has direct experimental access to the region inside the horizon, and so the spectrum of the phonons emitted there will be observable. Our results predict that this spectrum will be completely different from the one emitted outside the horizon. In particular, it will not be thermal.

\section{ACKNOWLEDGMENTS}

R. B. would like to thank T. Jacobson, R. Parentani, J. Steinhauer and S. Weinfurtner for discussion. A. F. acknowledges partial financial support by the Spanish Ministerio de Economía, Industria y Competitividad Grant No. FIS201784440-C2-1-P, the Generalitat Valenciana Project No. SEJI/ 2017/042 and the Severo Ochoa Excellence Center Project No. SEV-2014-0398. R. A. D. thanks the University of Valencia, where some of this work was done, for hospitality and acknowledges partial financial support from the Paul K. and Elizabeth Cook Richter Memorial Fund. This work was supported in part by the National Science Foundation under Grants No. PHY-1505875 and No. PHY-1912584 to Wake Forest University. 
[1] W. Unruh, Phys. Rev. Lett. 46, 1351 (1981).

[2] C. Barcelo, S. Liberati, and M. Visser, Living Rev. Relativity 8, 12 (2005).

[3] L. Pitaevskii and S. Stringari, Bose-Einstein Condensation (Oxford University, New York, 2003).

[4] L. J. Garay, J. R. Anglin, J. I. Cirac, and P. Zoller, Phys. Rev. Lett. 85, 4643 (2000).

[5] J. Macher and R. Parentani, Phys. Rev. A 80, 043601 (2009).

[6] A. Recati, N. Pavloff, and I. Carusotto, Phys. Rev. A 80, 043603 (2009).

[7] S. W. Hawking, Nature (London) 248, 30 (1974); Commun. Math. Phys. 43, 199 (1975).

[8] J. Steinhauer, Nat. Phys. 12, 959 (2016).

[9] J. R. M. de Nova, K. Golubkov, V. I. Kolobov, and J. Steinhauer, Nature (London) 569, 688 (2019).

[10] R. Balbinot, A. Fabbri, S. Fagnocchi, A. Recati, and I. Carusotto, Phys. Rev. A 78, 021603 (2008).
[11] I. Carusotto, S. Fagnocchi, A. Recati, R. Balbinot, and A. Fabbri, New J. Phys. 10, 103001 (2008).

[12] R Dudley, P. R. Anderson, A. Fabbri, and R. Balbinot, Phys. Rev. D 98, 124011 (2018).

[13] R. Balbinot, I. Carusotto, A. Fabbri, C. Mayoral, and A. Recati, Lect. Notes Phys. 870, 181 (2013).

[14] D. G. Boulware, Phys. Rev. D 11, 1404 (1975).

[15] W. Unruh, Phys. Rev. D 14, 870 (1976).

[16] P. R. Anderson, R. Balbinot, A. Fabbri, and R. Parentani, Phys. Rev. D 87, 124018 (2013).

[17] G. Jannes, P. Maïssa, T. G. Philbin, and G. Rousseaux, Phys. Rev. D 83, 104028 (2011).

[18] A. Coutant, A. Fabbri, R. Parentani, R. Balbinot, and P. R. Anderson, Phys. Rev. D 86, 064022 (2012).

[19] S. Corley and T. Jacobson, Phys. Rev. D 54, 1568 (1996). 